\title{
The influence of a single thiol group on the electronic and optical properties of the smallest diamondoid adamantane
}

\author{
Lasse Landt, ${ }^{1, \text { a) }}$ Matthias Staiger, ${ }^{1}$ David Wolter, ${ }^{1}$ Kathrin Klünder, ${ }^{1}$ Peter Zimmermann, ${ }^{1}$ \\ Trevor M. Willey, ${ }^{2}$ Tony van Buuren, ${ }^{2}$ Daniel Brehmer, ${ }^{3}$ Peter R. Schreiner, ${ }^{4}$ \\ Boryslav A. Tkachenko, ${ }^{4}$ Andrey A. Fokin, ${ }^{4}$ Thomas Möller, ${ }^{1}$ and Christoph Bostedt ${ }^{1}$ \\ ${ }^{1}$ Institut für Optik und Atomare Physik, Technische Universität Berlin, Eugene-Wigner-Bldg. EW 3-1, \\ Hardenbergstr. 36, 10623 Berlin, Germany \\ ${ }^{2}$ Condensed Matter and Materials Division, Lawrence Livermore National Laboratory, 7000 East Avenue, \\ Livermore, California 94550, USA \\ ${ }^{3}$ Stanford Synchrotron Radiation Lightsource, SLAC National Accelerator Laboratory, 2575 Sand Hill Road, \\ Menlo Park, California 94025, USA \\ ${ }^{4}$ Institute of Organic Chemistry, Justus-Liebig University, Heinrich-Buff-Ring 58, 35392 Giessen, Germany
}

(Received 14 October 2009; accepted 8 December 2009; published online 13 January 2010)

\begin{abstract}
At the nanoscale, the surface becomes pivotal for the properties of semiconductors due to an increased surface-to-bulk ratio. Surface functionalization is a means to include semiconductor nanocrystals into devices. In this comprehensive experimental study we determine in detail the effect of a single thiol functional group on the electronic and optical properties of the hydrogen-passivated nanodiamond adamantane. We find that the optical properties of the diamondoid are strongly affected due to a drastic change in the occupied states. Compared to adamantane, the optical gap in adamantane-1-thiol is lowered by $\sim 0.6 \mathrm{eV}$ and UV luminescence is quenched. The lowest unoccupied states remain delocalized at the cluster surface leaving the diamondoid's negative electron affinity intact. (C) 2010 American Institute of Physics.

[doi:10.1063/1.3280388]
\end{abstract}

\section{INTRODUCTION}

Nanocrystals possess novel properties due to quantum size effects that become relevant on the nanometer scale. To exploit these properties, however, one has to think of ways to include them in more macroscopic devices. Surface functionalization allows a viable means for attaching nanoparticles to various other entities such as biomolecules, organic semiconductors, or metal surfaces. ${ }^{1,2}$ Bonding to the latter is typically achieved via thiol functional groups, which form selfassembled monolayers (SAMs) on, e.g., Au or Ag surfaces. ${ }^{3,4}$ The surface of nanocrystals, due to an increased surface-tobulk ratio, plays a vital role for their electronic structure. ${ }^{5}$ The effect of different surface passivants has been the subject of several experimental and theoretical studies. ${ }^{5-8}$ Surface functionalization, i.e., the replacement of a single surface passivant with another, is also expected to have a considerable impact on the electronic structure. ${ }^{9}$ Experimental data, however, are scarce. From an applied viewpoint it is important to know exactly how functional groups for device fabrication such as thiols affect the nanocrystal properties. In the worst case, the functionalization may cause unintended changes to the relevant properties resulting in adverse effects or even prohibiting use for the designated application. In the ideal case, however, surface functionalization provides efficient means to intentionally modify the properties of nanocrystals in a controllable manner ${ }^{10}$ and exploit them for nanotechnology devices.

\footnotetext{
${ }^{\text {a) }}$ Author to whom correspondence should be addressed. Electronic mail: landt@physik.tu-berlin.de.
}

Diamondoids, hydrogen-passivated diamond fragments, are promising nanoscale materials. ${ }^{11}$ Their subnanometer size, their perfect bulk diamond structure, and their size and shape selectability make them appealing candidates for fundamental and applied research alike. Since the discovery of higher diamondoids in natural oil resources ${ }^{11}$ several experimental ${ }^{12-16}$ and theoretical studies ${ }^{17,18}$ have revealed numerous unique and partly unanticipated properties. These properties, such as shape dependent optical absorption, ${ }^{14}$ ultraviolet photoluminescence, ${ }^{15}$ or negative electron affinity (NEA),${ }^{17}$ reveal the great prospects that diamondoids bear for technological use ${ }^{19}$ and foster the growing interest in diamondoids as building blocks for nanotechnology.

This development has gained momentum within the last few years due to the successful preparation of surface modified diamondoids. ${ }^{20} \mathrm{~A}$ variety of diamondoids with different functional groups are now available ${ }^{21-24}$ and further ways to modify diamondoid properties have recently been realized ${ }^{25}$ or proposed. ${ }^{26,27}$ Most notably, thiolated higher diamondoids have been prepared. ${ }^{28}$ SAMs of adamantane-1-thiol on $\mathrm{Au}(111)$ surfaces have been studied extensively. ${ }^{29-31}$ They are less faulty than alkane SAMs and have, among others, been proposed as placeholder in intelligent self-assembly. ${ }^{30}$ Similar structures involving an adamantane cage unit and a thiol link have also been investigated. ${ }^{32-34}$ The availability of higher diamondoid thiols now allows for their simple inclusion into solid state devices via SAMs. ${ }^{35,36}$ Recent results on SAMs of diamondoid thiols, ${ }^{37}$ giving first experimental evidence of NEA, display their great technological potential. 
A series of experimental studies on pristine diamondoids has yielded fundamental insight into the physical processes governing semiconductors on the nanoscale. ${ }^{13-15,17}$ The chemical functionalization of diamondoids now allows extension of this series to experimentally quantify the effect of single functional groups and, more generally, assess the importance of a nanocrystal's surface composition as a whole. It is the deeper understanding of the influence of the surface chemistry that will ultimately enable us to properly exploit the physical properties of nanocrystals.

In this paper we examine the technologically interesting example of a thiol group attached to adamantane, ${ }^{30,31}$ the smallest diamondoid. ${ }^{11}$ We show that the optical properties of adamantane are strongly altered upon attachment of a single thiol group, including a shift of the optical gap of 0.6 $\mathrm{eV}$. As discussed in detail below, this can be explained mainly in terms of effects on the occupied states caused by a nonbonding electron lone pair of the thiol group. The lowest unoccupied molecular orbitals (LUMO), which are held responsible for the diamondoid's NEA, ${ }^{17}$ remain nearly unchanged. UV photoluminescence, found for adamantane, ${ }^{15}$ could not be detected for adamantane-1-thiol.

The presented study, combining several experimental techniques with quantum chemical calculations in a comprehensive investigation, elucidates the influence of a single functional group on this nanodiamond's electronic and optical properties. The paper is structured as follows. In Sec. II we will describe the experimental techniques that we employed to investigate adamantane-1-thiol. In addition to the experimental methods described below we performed density functional theory (DFT) computations also described in Sec. II. In Sec. III we present the results of the experiments in the same order as introduced in Sec. II. We compare the data recorded for adamantane-1-thiol to existing data for pristine adamantane. ${ }^{13,14,38,39}$ This comparison isolates the influence of the thiol functional group on individual properties of the diamondoid. In Sec. IV we discuss in detail the data presented in Sec. III. A summary is given in Sec. V.

\section{METHODS}

The purities of all samples used exceed 99\% and all measurements have been conducted on samples in the gas phase except for the Raman spectroscopy, which has been performed on a condensed sample (van-der-Waals crystal). The conducted gas phase experiments on high purity samples allow the determination of the inherent properties of the cluster without interaction with its surroundings or with other clusters. ${ }^{40}$ This makes the present data directly comparable to theory. ${ }^{14}$ The preparation of the samples has been described elsewhere. ${ }^{28}$

\section{A. X-ray photoelectron spectroscopy}

$\mathrm{X}$-ray photoelectron spectroscopy (XPS) is used to probe the energetic position of the core levels with respect to the vacuum level. The XPS data were recorded at beamline UE56/2-PGM-2 of the Berlin synchrotron facility BESSY II. The samples were evaporated into the vacuum chamber and the particle beam, guided by a nozzle, was crossed with monochromatized synchrotron light. We used an excitation energy of $331 \mathrm{eV}$. The resulting photoelectrons were then recorded by a Scienta SES-2002 hemispherical electron analyzer. The spectra were taken by scanning the kinetic energy of the electrons from $39-43 \mathrm{eV}$ in increments of $0.01 \mathrm{eV}$. Spectral resolution with the used beamline and detector settings was determined to be better than $125 \mathrm{meV}$. Prior to every measurement we acquired an Ar spectrum to which the data were later calibrated.

\section{B. Ultraviolet photoelectron spectroscopy}

Ultraviolet photoelectron spectroscopy (UPS) probes the valence states (highest occupied states) of the cluster. The UPS data were recorded using the same vacuum chamber/ detector setup described in Sec. II A. Instead of a synchrotron we used a helium lamp with an excitation energy of $21.22 \mathrm{eV}$ as the light source. The samples reached sufficient vapor pressure at room temperature and no additional heating was required. Kinetic energy was scanned from 10-14 $\mathrm{eV}$ in increments of $0.01 \mathrm{eV}$. Krypton gas served as calibrant and resolution with the used detector settings was determined to be better than $40 \mathrm{meV}$.

\section{Raman spectroscopy}

Raman spectroscopy is a standard tool to reveal vibrational modes of the investigated samples. The Raman spectra were taken using a Dilor LabRam spectrometer $(1800 \mathrm{l} / \mathrm{mm}$ grating). The $488 \mathrm{~nm}$ line of an Argon ${ }^{+}$laser was used as exciting radiation and the spectral resolution was $2 \mathrm{~cm}^{-1}$. Raman spectroscopy was performed on condensed samples under atmosphere. Prior to measurements, the setup and procedure were used to reproduce the known spectrum of adamantane $^{39}$ for calibration purposes.

\section{X-ray absorption spectroscopy}

$\mathrm{X}$-ray absorption spectroscopy (XAS) is used to gain information about the unoccupied states of the cluster. XAS measurements were conducted at beamline 10.1 at the Stanford Synchrotron Radiation Lightsource, SLAC National Accelerator Laboratory. ${ }^{41}$ The spectra were acquired using a simple gas cell consisting of two conductive plates above and below the incident beam, separated by about $1 \mathrm{~cm}$, with a bias between these plates of $100 \mathrm{~V}$. A thin aluminum window supported on a nickel grid separated the cell from the beamline. The cell was evacuated, purged multiple times with $\mathrm{N}_{2}$ gas, and filled with 20 mtorr $(0.03$ mbar $)$ of adamantane/adamantane-1-thiol. The absorption signal was the small current between these two plates while scanning the incident photon energy through the carbon K-edge. Signal normalization was achieved by dividing the signal by the electron yield current from an upstream gold grid. The spectrum of 20 mtorr $\mathrm{CO}_{2}$, acquired before and after each scan, was used to calibrate energy scales. This method produced adamantane spectra indistinguishable from previous experiments using a different detection scheme. ${ }^{13}$ The resolution was determined to be $0.05 \mathrm{eV}$. 


\section{E. Optical absorption spectroscopy}

Optical absorption reflects band-to-band transitions from the cluster's ground state into an electronically excited state. Optical absorption measurements were performed at the ultraviolet beamline I with the cluster physics endstation CLULU (Ref. 42) of the Hamburg synchrotron facility HASYLAB at DESY. For the absorption measurements a heatable, vacuum ultraviolet-transparent, custom built absorption cell was employed ${ }^{14}$ to handle the small sample quantities available. The photon energy was scanned from $5.0-11.2 \mathrm{eV}$. The photon energy resolution has been determined to be better than $20 \mathrm{meV}$ over the entire energy range. Second order and higher harmonics were suppressed using a LiF filter with an energy cutoff at $11.8 \mathrm{eV}$. The absorption data are normalized to the photon flux. For the absorption measurements adamantane-1-thiol was moderately heated $\left(\mathrm{T}_{\max }=310 \mathrm{~K}\right)$ to reach vapor pressures that yield sufficient signal intensities.

\section{F. Photoluminescence measurements}

Luminescence stems from the relaxation of an excited state to the ground state producing the emission of a photon. Luminescence measurements have been performed at the Berlin synchrotron facility BESSY II at beamline U125/2. The setup has been described in detail in a previous publication. ${ }^{15}$ In short, the incoming monochromatized pho-

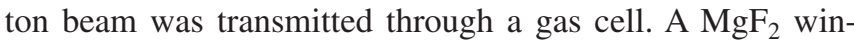
dow perpendicular to the incident beam allowed the detection of luminescence. We used a CsTe microchannel-plate detector, which is sensitive to wavelengths from 160 to 300 $\mathrm{nm}$. Luminescence measurements were performed at room temperature.

\section{G. Computational methods}

To help interpretation of the experimental data we performed ab initio DFT computations. We used the well established B3LYP functional with a 6-31G(d) basis set as implemented in the program package GAUSSIAN $03,{ }^{43}$ which is routinely used for the theoretical treatment of the electronic structure of nanoscale objects such as diamondoids. ${ }^{18,44}$ Application of larger basis sets to random diamondoid structures did not change the qualitative findings used in the discussion of the data. The results of the calculations will be used throughout the different sections. Next to electronic levels we have also computed the vibrational spectra shown in Figs. 3(b) and 3(c), which will be discussed in Sec. III C.

\section{RESULTS}

\section{A. C 1s core levels}

In Fig. 1 the spectra of the carbon 1s core level for adamantane-1-thiol and adamantane are shown. Both exhibit a steep incline on the lower binding energy side and a tail toward higher energies. This publication shall focus on the effects of a thiol group on the electronic properties and therefore on the differences of the two spectra. A more detailed interpretation of the core level spectra of adamantane and higher diamondoids will given in a later publication. ${ }^{45}$ While the appearance of the spectra is the same, the $\mathrm{C} 1 \mathrm{~s}$ levels of

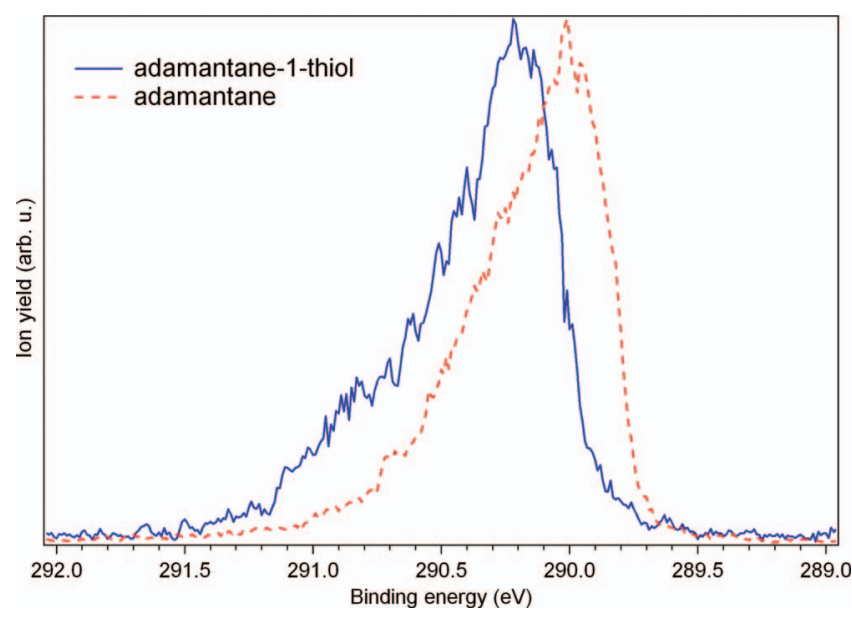

FIG. 1. C 1s core level spectra for adamantane-1-thiol and adamantane. Electrons from the $\mathrm{C}$ 1s core levels are more strongly bound in the thiol by $\sim 0.2 \mathrm{eV}$. Spectra have been scaled to same height to simplify comparison.

the thiol are shifted by $0.2 \mathrm{eV}$ to higher binding energies. This shift is due to the transfer of electron density from the parent molecule to the highly electronegative thiol group. The electron transfer results in lower electron density in the parent cluster and thus in more strongly bound electrons. Both spectra have been scaled to the same height for the sake of comparability and the structure in the high energy tail of the adamantane-1-thiol is due to lower count rates, i.e., increased signal-to-noise ratio.

\section{B. Occupied states}

In Fig. 2 the photoelectron spectra of adamantane (bottom) and adamantane-1-thiol (top) are displayed. For the discussion of the data it proves useful to split the thiol spectrum in two parts, a band structure of electronic states starting at binding energies of $9.5 \mathrm{eV}$ and a broad peak centered around

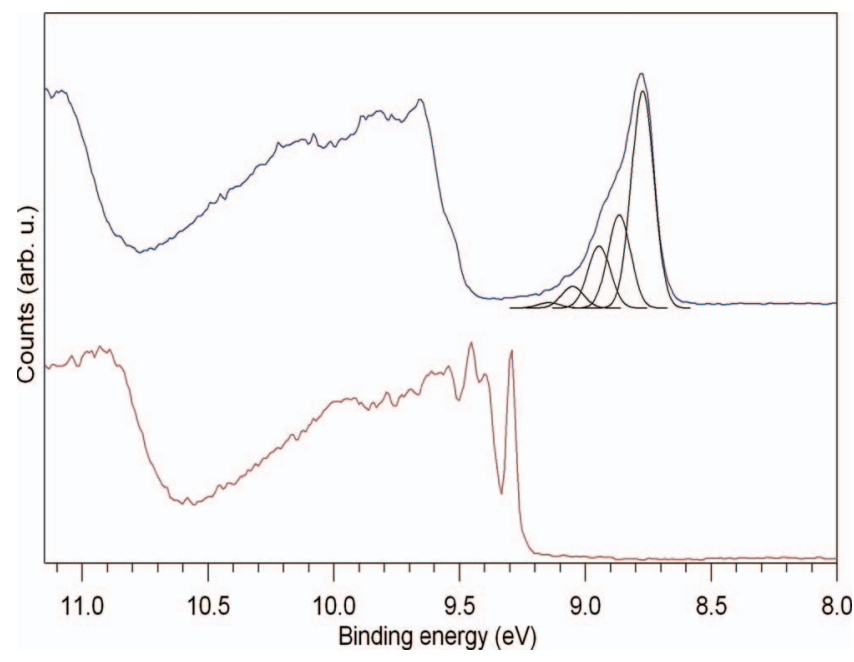

FIG. 2. Photoelectron spectra for adamantane-1-thiol (top) and adamantane (bottom). (Spectra have been scaled to same height to simplify comparison.) The thiol spectrum still contains the adamantane spectrum, even though it appears shifted to higher binding energies by about $0.2 \mathrm{eV}$ and the vibrational structure is attenuated. An additional density of states arises for the thiol around $8.8 \mathrm{eV}$. It lowers the ionization potential by $0.58 \mathrm{eV}$ compared to the unmodified cluster (Ref. 48). The peak has been fitted with five Gaussian peaks to resolve vibronic structure. 
TABLE I. Electronic key figures of adamantane-1-thiol compared to literature values for adamantane. $\Delta$ gives the difference between values for adamantane-1-thiol and adamantane. Negative values correspond to a decrease in the corresponding values upon thiolation, positive values to an increase. All values are given in $\mathrm{eV}$.

\begin{tabular}{lccr}
\hline \hline & $\begin{array}{c}\text { Adamantane-1-thiol } \\
\left(\mathrm{C}_{10} \mathrm{H}_{15}-\mathrm{SH}\right)\end{array}$ & $\begin{array}{c}\text { Adamantane } \\
\left(\mathrm{C}_{10} \mathrm{H}_{16}\right)\end{array}$ & \multicolumn{1}{c}{$\Delta$} \\
\hline Optical gap & $5.85 \pm 0.06$ & $6.49 \pm 0.03^{\mathrm{a}}$ & -0.64 \\
Adiabatic IP & $8.65 \pm 0.04$ & $9.23 \pm 0.11^{\mathrm{b}}$ & -0.58 \\
Cond. band edge & $286.6 \pm 0.1$ & $286.5 \pm 0.1^{\mathrm{c}}$ & 0.1 \\
Core level & $289.9 \pm 0.1$ & $289.7 \pm 0.1$ & 0.2 \\
\hline \hline
\end{tabular}

${ }^{\mathrm{a}}$ Reference 14.

${ }^{\mathrm{b}}$ Reference 48 .

${ }^{\mathrm{c}}$ Reference 13.

$8.8 \mathrm{eV}$. These two parts are separated in the spectrum by a gap of a few hundred meV (located around $9.2 \mathrm{eV}$ binding energy) where no electronic levels are present.

The part of the thiol spectrum beyond binding energies of $9.3 \mathrm{eV}$ can be superimposed on the adamantane spectrum. This shows that these electronic states belong to the diamondoid parent molecule and are not greatly affected by the thiolation. Only the vibronic resonances ${ }^{38}$ at the highest occupied states in the adamantane spectrum are attenuated in the adamantane thiol. This part of the thiol spectrum is also shifted to higher binding energy by approximately $0.2 \mathrm{eV}$. This is the same effect that is observed in the core level spectra in Fig. 1, which is due to redistribution of electron density upon thiol attachment. The electron depletion in the diamondoid part leads to the formation of a local positive charge (polarization) thus increasing the binding energy. The good agreement with the adamantane spectrum is not selfevident because the thiolation breaks down the symmetry of the cluster from $\mathrm{T}_{d}$ for adamantane to $\mathrm{C}_{s}$ for adamantane-1thiol. This revokes the symmetry-induced degeneracy of the electronic levels found in adamantane. However, the resemblance of the spectral features implies that the orbitals remain quasidegenerate in adamantane-1-thiol.

By contrast, the second part of the thiol spectrum, the broad peak centered around $8.8 \mathrm{eV}$, possesses no analog in the adamantane spectrum. By analogy with closely related molecules, such as alkane thiols, ${ }^{46,47}$ this additional density of states can be ascribed to the $n_{S}^{\perp}$ nonbonding out-of-plane electron lone pair from the thiol group. This "thiol state" lowers the IP compared to adamantane ${ }^{48}$ by $0.58-8.65 \mathrm{eV}$. Listed in Table I are the adiabatic IPs, which are determined as intersection of a tangent to the graph and the baseline. ${ }^{48}$ Our quantum chemical calculations of the molecular orbitals confirm that the thiol state, despite its broadness, is indeed due to only a single electronic level. The thiol state is broadened by vibronic transitions resolved in Fig. 2 by five Gaussian peaks. These peaks have an energy spacing of approximately $90 \mathrm{meV}$, as discussed in more detail in Sec. IV. The predominance of the first Gaussian peak at the low energy side of the thiol state suggests that the adiabatic transition is by far the strongest in accordance with the nonbonding character of the orbital. To identify the excited vibrational modes we employed Raman spectroscopy.

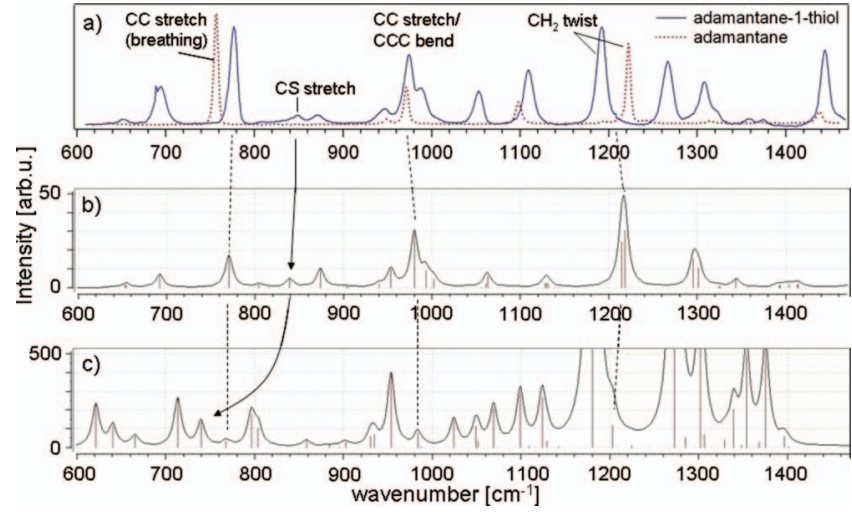

FIG. 3. Experimental Raman spectrum for the adamantane-1-thiol (a) in comparison to the computed spectra for the neutral cluster (b) and the cation (c). In (a) the measured spectrum for adamantane is added for comparison (dotted line). The Raman peaks connected by arrows correspond to a vibrational mode of mainly SC stretch character. This mode has an energy of 92 $\mathrm{meV}$ in the cluster ion, in excellent agreement with the vibronic structure shown in Fig. 2. Note that the Raman intensities for the neutral cluster and the ion are plotted over different scales.

\section{Vibronic structure}

Figure 3 compares the measured Raman spectrum of adamantane-1-thiol (a) to the spectra computed for neutral cluster (b) and the cation (c). The latter is added because the UPS data presented in Sec. III B that we strive to explain reflect the ionic final state of the cluster. This means that the data in Fig. 2, which will be discussed in Sec. IV, contain the vibrational modes of the cluster ion. For the methane thiol optimizing the ion geometry prior to computation of the Raman modes yields far better agreement with experimental data than the ion in the ground state geometry. ${ }^{46}$ Therefore the Raman spectrum of the adamantane-1-thiol cation has been computed in its optimized geometry. The Raman spectrum of adamantane has been added to Fig. 3(a) for comparison.

We find good agreement between the measured (a) and the computed Raman spectrum (b) for adamantane-1-thiol. In the wave number region below $1000 \mathrm{~cm}^{-1}$ differences in frequencies generally remain within a few $\mathrm{cm}^{-1}$. Based on the agreement of the measured and the computed spectrum of the neutral thiol cluster we assume similar reliability for the computed Raman spectrum of the adamantane-1-thiol cation. Most vibrational modes that have been assigned in adamantane $^{39}$ are not severely affected by thiolation. The symmetric carbon-carbon (CC) stretch (breathing) mode of adamantane, which is found at $757 \mathrm{~cm}^{-1}$, e.g., is found for adamantane-1-thiol at $776 \mathrm{~cm}^{-1}$ in the measured and $770 \mathrm{~cm}^{-1}$ in the computed Raman spectrum. The same holds true for most of the other vibrational modes not directly involving the thiol group. The new modes that arise in the adamantane-1-thiol spectrum with respect to adamantane, depicted as dotted line in Fig. 3(a), all exhibit the significant participation of the thiol group.

\section{Unoccupied states}

The XAS data for both thiolated (top) and pristine adamantane (bottom) are displayed in Fig. 4. The XAS data 


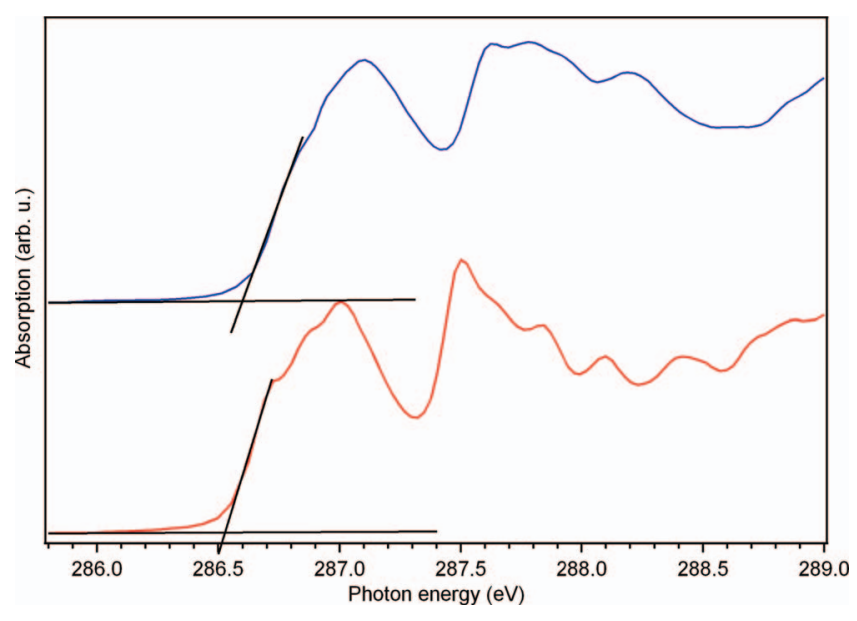

FIG. 4. X-ray absorption data for adamantane-1-thiol (top) compared with pristine adamantane (bottom). The data reveal that the unoccupied states remain nearly unchanged upon thiolation with only a small shift of $\sim 0.1 \mathrm{eV}$ to higher energy. This suggests that the surface nature of the LUMO (Ref. 13) remains relatively unchanged. The spectra have been scaled to same height to simplify comparison.

show that thiolation induces only minor variations in the unoccupied states of the cluster. Upon thiol attachment the absorption onset as well as the two main resonances, which are found at 287.0 and $287.5 \mathrm{eV}$ for adamantane, are slightly shifted to higher energies by $\sim 0.1 \mathrm{eV}$. In an earlier study these first two resonances could be linked to the $\mathrm{CH}$ - and the $\mathrm{CH}_{2}$-environment of the diamondoid's surface atoms showing that the LUMO is determined by the hydrogen surface and is delocalized at the outside of the cluster. ${ }^{13}$ The data for adamantane-1-thiol show that upon thiolation the unoccupied states remain largely unchanged in energy as well as in their delocalized nature. ${ }^{12,13,17}$ The latter is again confirmed by our electronic structure computations showing the lowest unoccupied states to be distributed around the cluster's hydrogen shell.

\section{E. Optical transitions}

In Fig. 5 the optical spectra of the adamantane-1-thiol (top) and the pristine diamondoid (bottom) are compared. The absorption energies lie in the same regime. For the adamantane thiol a broad resonance is marking the absorption onset with an optical gap of $5.85 \mathrm{eV}$. This corresponds to a lowering of the optical gap of adamantane, which was measured to be $6.49 \mathrm{eV}^{14}$ by $0.64 \mathrm{eV}$ due to thiolation. The spectrum of adamantane exhibits numerous very sharp peaks, which have been attributed to vibronic excitations and Rydberg states ${ }^{49}$ while the thiol spectrum is mostly smooth with only a few, rather broad resonances. The lack of structure in the thiol optical spectrum also goes hand in hand with the disappearance of vibronic structure observed in Fig. 2 and discussed in Sec. III C. The attachment of a thiol group leads to the lower $\mathrm{C}_{s}$ symmetry compared to $\mathrm{T}_{d}$ adamantane. Symmetry also implies that for the thiol, unlike adamantane, no restrictions due to dipole selection rules apply to the optical transitions. This is, next to the disappearance of Rydberg states, another reason for disappearance of sharp transitions. Symmetry constraints for the optical transitions in

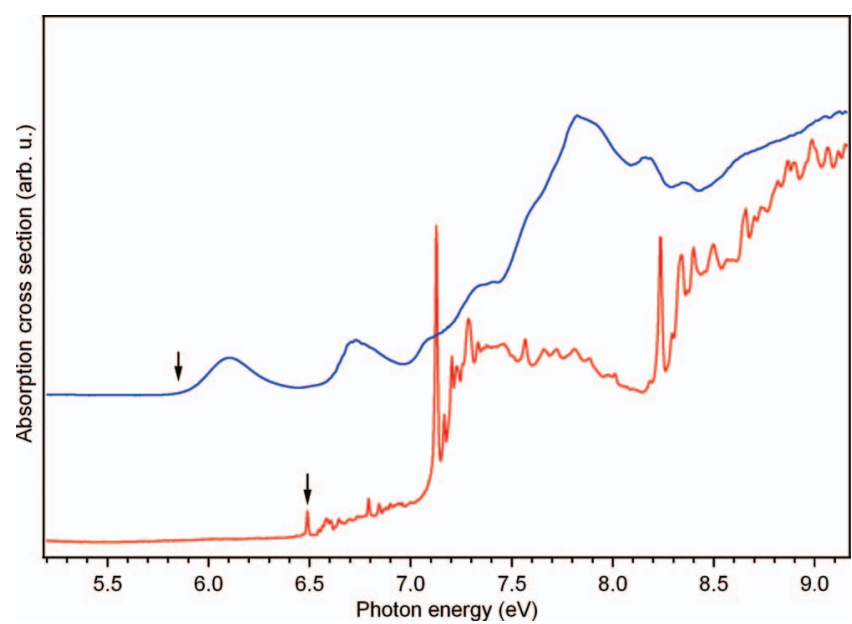

FIG. 5. Optical absorption of adamantane-1-thiol (top) compared to that of pristine adamantane (bottom). Most obvious is the disappearance of the sharp resonances in the adamantane spectrum. Also the optical gap, as defined in Ref. 14 (arrows), is lowered by $0.64 \mathrm{eV}$. The spectra have been scaled to similar height.

adamantane, however, do not concern the lowest transitions ${ }^{14}$ and are therefore not responsible for the differences found in the optical gaps observed in Fig. 5 and listed in Table I.

\section{F. Photoluminescence}

For adamantane-1-thiol we used the same setup that was used to measure the luminescence of adamantane ${ }^{15}$ to check for photoluminescence in the energy range from 4.1 to 7.7 $\mathrm{eV}$. No photoluminescence has been detected upon excitation between 5.6 and $8.8 \mathrm{eV}$, which covers band-to-band transitions from the optical gap to the IP (comp. Table I). This means the UV luminescence found for adamantane ${ }^{15}$ is quenched by at least two orders of magnitude due to the attachment of a thiol group.

\section{DISCUSSION}

In Sec. III we have reported the experimental data for the $\mathrm{C}$ 1s core level, the highest occupied and lowest unoccupied electronic states and the optical transitions between them, and the Raman data. In this section the $\mathrm{x}$ ray and ultraviolet photoelectron, x-ray absorption, Raman, and optical data are combined to give deeper insight into how the addition of a thiol group changes the properties of adamantane. Figure 6 depicts a schematic graphical summary of the changes in the electronic structure.

Particularly poignant are the observed changes in the optical properties and their discussion benefits from the knowledge of the band edge data introduced above. In the following the changes in optical properties, i.e., band-toband transitions, are compared to changes in the energy gap between highest occupied and lowest unoccupied molecular orbitals (HOMO-LUMO gap), which are reflected by the band edge measurements in Secs. III B and III D.

Considering the spectra in Figs. 2 and 4 the changes in the optical response primarily arise from changes in the occupied states. The redshift of $0.64 \mathrm{eV}$ of the optical gap can be accounted for by the additional electron lone-pair state 


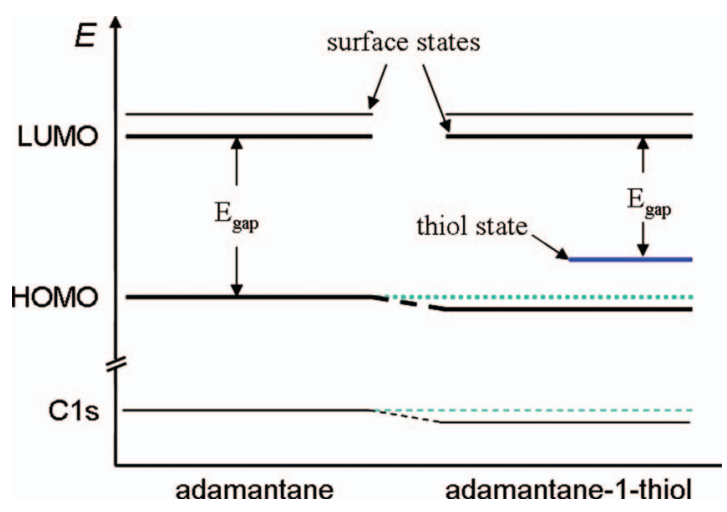

FIG. 6. Schematic drawing of the electronic levels of adamantane and adamantane-1-thiol. In the functionalized diamondoid the $\mathrm{S}$ electron lone pair gives rise to a "thiol state" constituting the HOMO of adamantane-1thiol and, effectively, lowering the gap by $\sim 0.6 \mathrm{eV}$.

that arises for the thiol $0.58 \mathrm{eV}$ above the band edge of adamantane (Fig. 2). This lowering of the optical gap leads to a gap value for adamantane-1-thiol that is comparable to penta- and hexamantanes, i.e., nanodiamonds with five and six cage units, respectively. ${ }^{14}$ However, it is of a different nature because it takes place between the surface dominated LUMO and the HOMO, which is now given by the electron lone-pair state of the thiol group. The first two noticeable spectral features in the optical absorption, shown in Fig. 5, are centered around 6.1 and $6.7 \mathrm{eV}$ with an energy spacing of $0.6 \mathrm{eV}$. This energy spacing is in good agreement with the first two manifolds of resonances in the XAS spectrum in Fig. 4. Assuming that the first two resonances in the optical spectrum in Fig. 5 are transitions from the HOMO, the broadness of these two peaks can be explained with the convolution of the thiol state and the respective features in the XAS spectrum shown in Fig. 2. A "main resonance" can be located around $7.8 \mathrm{eV}$ for the thiol. This resonance appears in the spectrum of the pristine diamondoid somewhat disguised by Rydberg resonances and shifted to lower energies by some $300-400 \mathrm{meV}$. This corresponds to the shift of more than $0.2 \mathrm{eV}$, which has been observed in the photoelectron data in Secs. III A and III B (Fig. 2) and the additional small shift in the unoccupied states (Fig. 4). The discussion above shows that most of the changes that occur in the optical response and the optical gap can be fairly well reconstructed from comparison with occupied and unoccupied states and the HOMO-LUMO gap, respectively.

Preliminary theoretical work ${ }^{50}$ as well as some earlier studies on alkane thiols ${ }^{51,52}$ suggest that there might be weak optical transitions occurring at energies around $5.5 \mathrm{eV}$. Our data, however, do not identify such transitions. In fact, the absorption cell setup we used in this work has been shown to be very sensitive also revealing minor optical transitions. ${ }^{14}$ The present data only leave room for transitions that would have to be around 100 times weaker than the transition found for adamantane-1-thiol at $6.1 \mathrm{eV}$. It remains an open question if this disagreement is due to the complete absence of the predicted transitions or oscillator strengths below the detection limit. Further research featuring detailed theoretical in- vestigation of presumable optical transitions in the energy range of 5-6 eV will be conducted to clarify this issue and published in a follow-up paper. ${ }^{53}$

The results in Sec. III F, namely, the lack of detectable photoluminescence for adamantane-1-thiol, suggest that UV photoluminescence found in adamantane ${ }^{15}$ is quenched by the attachment of a thiol group. In Sec. III B we saw that the electron lone pair of the thiol group gives rise to an additional density of states above the adamantane HOMO. Because luminescence typically occurs due to transitions from the LUMO to the HOMO the altered nature of the HOMO is to be held accountable for the disappearance of the luminescence. Functional groups that leave intact both HOMO and LUMO might be suitable to exploit the luminescence properties of diamondoids in technological devices.

In Sec. III B, presenting the results for the valence states, we have tentatively assigned the shoulder of the additional peak in the adamantane-1-thiol spectrum in Fig. 2 to vibrational progressions. With the help of the supporting Raman data from Sec. III C we now want to underpin and specify the assignment made. The five Gaussian peaks used to fit the thiol state in Fig. 2 have an almost equidistant energy spacing of approximately $90 \mathrm{meV}$. The Raman peak in the spectrum computed for the ionic cluster that comes the closest is a mode of medium intensity at $739 \mathrm{~cm}^{-1}(92 \mathrm{meV})$. The computations show that this Raman signal belongs to a sulfur-carbon (SC) stretch mode. The same SC stretch mode is found in the neutral cluster at considerably higher wave numbers, dropping from $839 \mathrm{~cm}^{-1}$ in the neutral cluster to $739 \mathrm{~cm}^{-1}$ in the cluster ion (compare arrow in Fig. 3). This drastic change indicates that the SC stretch mode is strongly excited upon ionization. Next to the match in energy this is additional evidence that the SC stretch mode is responsible for the shoulder of the thiol peak in the PE spectrum of adamantane-1-thiol.

In Sec. III D we have seen that, unlike the HOMO, the LUMO of adamantane remains almost completely unaffected by the surface functionalization. This refers to the energy of the electronic states as well as to their delocalization at the nanodiamond's surface. The present data therefore suggest that properties of diamondoids that are intimately linked to the particular nature of their LUMO will withstand thiolation. This is the case for the technologically relevant NEA, which has been predicted for pristine diamondoids ${ }^{17}$ and has recently been verified experimentally for SAMs of [121] tetramantane-6-thiols. ${ }^{37,54}$ However, properties involving the LUMO and any additional electronic levels (e.g., the HOMO) may not persist, as seen in the case of photoluminescence.

\section{SUMMARY}

We have quantified the effects of a single thiol functional group on the electronic and optical properties of adamantane, the smallest diamondoid. Investigating adamantane-1-thiol we found that drastic changes occur in the optical properties of this hydrogen-passivated nanodiamond upon thiolation. The optical gap is reduced by $\sim 0.6 \mathrm{eV}$ and luminescence is quenched. Employing a variety of spectroscopic techniques 
we could show that these changes are due to an additional density of states that arises from the $\mathrm{S}$ electron lone pair that functions as a deep lying donor level (Fig. 6). In contrast, we find that only minor changes occur in the unoccupied states and that the surface nature of the LUMO remains intact. This finding is coherent with the recently found NEA for SAMs of diamondoid thiols. The present results demonstrate the significance of a single functional group by isolating and quantifying its influence on the electronic structure of a small nanodiamond. They are a step toward a more detailed understanding of the impact of surface chemistry.

\section{ACKNOWLEDGMENTS}

The authors are thankful to C. Thomsen and J. Maultzsch for access to the Raman spectroscopy setup, D. Heinrich for help with taking the Raman spectra, and to A. Kulesza, R. Mitrić, and V. Bonačić-Koutecký for discussion on the optical spectra. L.L. acknowledges support from the Studienstiftung des Deutschen Volkes. T.M.W. acknowledges funding from the UCOP management fee grant "carbon nanostructures." This work has been supported by the DFG under Grant No. BO3169/1-1.

${ }^{1}$ W. J. Parak, D. Gerion, D. Zanchet, A. S. Woerz, T. Pellegrino, C. Micheel, S. C. Williams, M. Seitz, R. E. Bruehl, Z. Bryant, C. Bustamante, C. R. Bertozzi, and A. P. Alivisatos, Chem. Mater. 14, 2113 (2002).

${ }^{2}$ M. F. Calhoun, J. Sanchez, D. Olaya, M. E. Gershenson, and V. Podzorov, Nature Mater. 7, 84 (2008).

${ }^{3}$ J. C. Love, L. A. Estroff, J. K. Kriebel, R. G. Nuzzo, and G. M. Whitesides, Chem. Rev. (Washington, D.C.) 105, 1103 (2005).

${ }^{4}$ S. K. Arya, P. R. Solanki, M. Datta, and B. D. Malhotra, Biosens. Bioelectron. 24, 2810 (2009).

${ }^{5}$ A. Puzder, A. J. Williamson, J. C. Grossmann, and G. Galli, Phys. Rev. Lett. 88, 097401 (2002).

${ }^{6}$ C. Bostedt, T. van Buuren, T. M. Willey, and L. J. Terminello, Appl. Phys. Lett. 85, 5334 (2004).

${ }^{7}$ Q. S. Li, R. Q. Shang, S. T. Lee, T. A. Niehaus, and T. Frauenheim, J. Chem. Phys. 128, 244714 (2008).

${ }^{8}$ L. E. Ramos, J. Furthmüller, and F. Bechstedt, Appl. Phys. Lett. 87, 143113 (2005).

${ }^{9}$ A. Puzder, A. J. Williamson, J. C. Grossmann, and G. Galli, J. Chem. Phys. 117, 6721 (2002).

${ }^{10}$ D. J. Norris, A. L. Efros, and S. C. Erwin, Science 319, 1776 (2008).

${ }^{11}$ J. E. Dahl, S. G. Liu, and R. M. K. Carlson, Science 299, 96 (2003).

${ }^{12}$ Y. Wang, E. Kioupakis, X. Lu, D. Wegner, R. Yamachika, J. E. Dahl, R. M. K. Carlson, S. G. Louie, and M. F. Crommie, Nature Mater. 7, 38 (2008).

${ }^{13}$ T. M. Willey, C. Bostedt, T. van Buuren, J. E. Dahl, S. G. Liu, R. M. K. Carlson, L. J. Terminello, and T. Möller, Phys. Rev. Lett. 95, 113401 (2005).

${ }^{14}$ L. Landt, K. Klünder, J. E. Dahl, R. M. K. Carlson, T. Möller, and C. Bostedt, Phys. Rev. Lett. 103, 047402 (2009).

${ }^{15}$ L. Landt, W. Kielich, D. Wolter, M. Staiger, A. Ehresmann, T. Möller, and C. Bostedt, Phys. Rev. B 80, 205323 (2009).

${ }^{16}$ T. M. Willey, C. Bostedt, T. van Buuren, J. E. Dahl, S. G. Liu, R. M. K. Carlson, R. W. Meulenberg, E. J. Nelson, and L. J. Terminello, Phys. Rev. B 74, 205432 (2006).

${ }^{17}$ N. D. Drummond, A. J. Williamson, R. J. Needs, and G. Galli, Phys. Rev. Lett. 95, 096801 (2005).

${ }^{18}$ A. A. Fokin and P. R. Schreiner, Mol. Phys. 107, 823 (2009).

${ }^{19}$ A. P. Marchand, Science 299, 52 (2003).

${ }^{20}$ H. Schwertfeger, A. A. Fokin, and P. R. Schreiner, Angew. Chem., Int. Ed. 47, 1022 (2008).

${ }^{21}$ P. R. Schreiner, N. A. Fokina, B. A. Tkachenko, H. Hausmann, M. Serafin, J. E. P. Dahl, S. Liu, R. M. K. Carlson, and A. A. Fokin, J. Org. Chem. 71, 6709 (2006)

${ }^{22}$ A. A. Fokin, P. R. Schreiner, N. A. Fokina, B. A. Tkachenko, H. Haus- mann, M. Serafin, J. E. P. Dahl, S. Liu, and R. M. K. Carlson, J. Org. Chem. 71, 8532 (2006).

${ }^{23}$ A. A. Fokin, E. D. Butova, L. V. Chernish, N. A. Fokina, J. E. P. Dahl, R. M. K. Carlson, and P. R. Schreiner, Org. Lett. 9, 2541 (2007).

${ }^{24}$ H. Schwertfeger, C. Würtele, H. Hausmann, J. E. P. Dahl, R. M. K. Carlson, A. A. Fokin, and P. R. Schreiner, Adv. Synth. Catal. 351, 1041 (2009).

${ }^{25}$ A. A. Fokin, T. S. Zhuk, A. E. Pashenko, P. O. Dral, P. A. Gunchenko, J. E. P. Dahl, R. M. K. Carlson, T. V. Koso, M. Serafin, and P. R. Schreiner, Org. Lett. 11, 3068 (2009).

${ }^{26}$ F. Marsusi and K. Mirabbaszadeh, J. Phys.: Condens. Matter 21, 215303 (2009).

${ }^{27}$ J. C. Garcia, J. F. Justo, W. V. M. Machado, and L. V. C. Assali, Phys. Rev. B 80, 125421 (2009).

${ }^{28}$ B. A. Tkachenko, N. A. Fokina, L. V. Chernish, J. E. P. Dahl, S. Liu, R. M. K. Carlson, A. A. Fokin, and P. R. Schreiner, Org. Lett. 8, 1767 (2006).

${ }^{29}$ T. J. Mullen, P. Zhang, C. Srinivasan, M. W. Horn, and P. S. Weiss, J. Electroanal. Chem. 621, 229 (2008).

${ }^{30}$ A. A. Dameron, L. F. Charles, and P. S. Weiss, J. Am. Chem. Soc. 127, 8697 (2005).

${ }^{31}$ A. A. Dameron, J. R. Hampton, R. K. Smith, T. J. Mullen, S. D. Gillmor, and P. S. Weiss, Nano Lett. 5, 1834 (2005).

${ }^{32}$ S. Fujii, U. Akiba, and M. Fujihira, J. Am. Chem. Soc. 124, 13629 (2002).

${ }^{33}$ T. Kitagawa, Y. Idomoto, H. Matsubara, D. Hobara, T. Kakiuchi, T. Okazaki, and K. Komatsu, J. Org. Chem. 71, 1362 (2006).

${ }^{34}$ M. Kim, N. Hohmann, E. I. Morin, T. A. Daniel, and P. S. Weiss, J. Phys. Chem. A 113, 3895 (2009).

${ }^{35}$ T. M. Willey, J. D. Fabbri, J. R. I. Lee, P. R. Schreiner, A. A. Fokin, B. A. Tkachenko, N. A. Fokina, J. E. Dahl, R. M. K. Carlson, A. L. Vance, W. Yang, L. J. Terminello, T. van Buuren, and N. A. Melosh, J. Am. Chem. Soc. 130, 10536 (2008).

${ }^{36}$ W. Zhang, B. Gao, J. Yang, V. Carvetta, and Y. Luo, J. Chem. Phys. 130, 054705 (2009).

${ }^{37}$ W. L. Yang, J. D. Fabbri, T. M. Willey, J. R. I. Lee, J. E. Dahl, R. M. K. Carlson, P. R. Schreiner, A. A. Fokin, B. A. Tkachenko, N. A. Fokina, W. Meevasana, N. Mannella, K. Tanaka, X. J. Zhou, T. van Buuren, M. A. Kelly, Z. Hussain, N. A. Melosh, and Z.-X. Shen, Science 316, 1460 (2007).

${ }^{38}$ W. Schmidt, Tetrahedron 29, 2129 (1973).

${ }^{39}$ J. Filik, J. N. Harvey, N. L. Allan, P. W. May, J. E. P. Dahl, S. Liu, and R. M. K. Carlson, Spectrochim. Acta, Part A 64, 681 (2006).

${ }^{40}$ C. Bostedt, T. van Buuren, T. M. Willey, N. Franco, L. J. Terminello, C. Heske, and T. Möller, Appl. Phys. Lett. 84, 4056 (2004).

${ }^{41}$ V. P. Karpenko, J. H. Kinney, S. Kulkarni, K. Neufeld, and C. Poppe, Rev. Sci. Instrum. 60, 1451 (1989).

${ }^{42}$ R. Karnbach, M. Joppien, J. Stapelfeldt, J. Wörmer, and T. Möller, Rev. Sci. Instrum. 64, 2838 (1993).

${ }^{43}$ M. J. Frisch, G. W. Trucks, H. B. Schlegel et al., Gaussian 03, Revision C.02, Gaussian, Inc., Wallingford, CT, 2004.

${ }^{44}$ A. J. Lu, B. C. Pan, and J. G. Han, Phys. Rev. B 72, 035447 (2005).

${ }^{45}$ K. Klünder, L. Landt, T. M. Willey, T. van Buuren, J. E. Dahl, R. M. K. Carlson, T. Möller, and C. Bostedt (in preparation).

${ }^{46}$ D. Frost, F. Hering, A. Katrib, C. McDowell, and R. McLean, J. Phys. Chem. 76, 1030 (1972).

${ }^{47}$ K. Ohno, K. Imal, S. Matmoto, and Y. Harada, J. Phys. Chem. 87, 4346 (1983).

${ }^{48}$ K. Lenzke, L. Landt, M. Hoener, H. Thomas, J. E. Dahl, S. G. Liu, R. M. K. Carlson, T. Möller, and C. Bostedt, J. Chem. Phys. 127, 084320 (2007).

${ }^{49}$ J. W. Raymonda, J. Chem. Phys. 56, 3912 (1972).

${ }^{50}$ A. Kulesza, R. Mitrić, and V. Bonačić-Koutecký (private communication).

${ }^{51}$ G. L. Vaghjiani, J. Chem. Phys. 99, 5936 (1993).

${ }^{52}$ L. B. Clark and W. T. Simpson, J. Chem. Phys. 43, 3666 (1965).

${ }^{53}$ L. Landt, D. Wolter, C. Bostedt, T. Möller, R. Mitrić, J. E. Dahl, R. M. K. Carlson, P. R. Schreiner, A. A. Fokin, A. Kulesza, and V. BonacićKoutecký (submitted).

${ }^{54}$ W. A. Clay, Z. Liu, W. L. Yang, J. D. Fabbri, J. E. Dahl, R. M. K. Carlson, Y. Sun, P. R. Schreiner, A. A. Fokin, B. A. Tkachenko, N. A. Fokina, P. A. Pianetta, N. Melosh, and Z.-X. Shen, Nano Lett. 9, 57 (2009). 